\title{
Lumbosacral plexus delineation, dose distribution, and its correlation with radiation-induced lumbosacral plexopathy in cervical cancer patients
}

This article was published in the following Dove Press journal:

OncoTargets and Therapy

23 December 2014

Number of times this article has been viewed

\author{
Mutahir Tunio' \\ Mushabbab Al Asiri' \\ Yasser Bayoumi ${ }^{2}$ \\ Ali Abdullah O Balbaid' \\ Majid AlHameed ${ }^{3}$ \\ Stanciu Laura Gabriela' \\ Ahmad Amir O Ali' \\ 'Radiation Oncology, Comprehensive \\ Cancer Center, King Fahad \\ Medical City, Riyadh, Saudi Arabia; \\ ${ }^{2}$ National Cancer Institute, Cairo \\ University, Cairo, Egypt; ${ }^{3}$ Neurology, \\ Comprehensive Cancer Center, \\ King Fahad Medical City, Riyadh, \\ Saudi Arabia
}

Background: To evaluate the dose distribution to the lumbosacral plexus (LSP) and its correlation with radiation-induced lumbosacral plexopathy (RILSP) in patients with cervical cancer treated with intensity-modulated radiotherapy (IMRT) and high-dose-rate brachytherapy.

Materials and methods: After meeting eligibility criteria, 50 patients with cervical cancer were selected who were treated with IMRT and high-dose-rate brachytherapy, and the LSP was contoured. Mean volume; percentages of LSP volume absorbing 40, 50, 55, and 60 Gy (V30, V40, V50, V55, and V60) and point doses (P1, P2, P3, P4, P5, P6, P7, P8, P9, and P10); and RILSP incidence were calculated.

Results: At 60 months of follow-up, four patients (8\%) were found to have grade 2/3 RILSP. The mean maximal LSP dose in patients with RILSP was 59.6 Gy compared with 53.9 Gy in patients without RILSP (control; $P=0.04$ ). The mean values of V40, V50, V55, and V60 in patients with RILSP versus control were $61.8 \%$ versus $52.8 \%, 44.4 \%$ versus $27.7 \%, 8.0 \%$ versus $0.3 \%$ and $1.8 \%$ versus $0 \%$, respectively ( $P=0.01,0.001,0.001$, and 0.001 , respectively).

Conclusion: The delineation of the LSP during IMRT planning may reduce the risk for RILSP. The mean values of V40, V50, V55, and V60 for LSP should be less than 55\%, 30\%, 5\%, and $0.5 \%$, respectively; however, further studies are warranted.

Keywords: cervical cancer, intensity-modulated radiation therapy, lumbosacral plexus delineation, radiation-induced lumbosacral plexopathy, dosimetric analysis

\section{Introduction}

Radiation-induced lumbosacral plexopathy (RILSP) in gynecologic cancers is a rare but extremely serious complication of pelvic irradiation. The reported frequency of RILSP ranges from $1.3 \%$ to $6.67 \%$, and the clinical course is associated with different degrees of bilateral lower limb pain, numbness, weakness, paresis or paralysis, and rarely, urinary or fecal incontinence leading to poor quality of life. ${ }^{1-3}$ However, the true incidence of RILSP is underestimated because it is not commonly evaluated by radiation oncologists, and symptoms are often overlooked, given the prevalence of lower back pain. ${ }^{4}$ In addition, the lumbosacral plexus (LSP) is not routinely contoured in practice for patients with gynecologic cancer receiving intensity-modulated radiation therapy (IMRT), which may lead to dose dumping, with higher than expected doses seen in the LSP because it is not specified as an organ at risk. ${ }^{5}$

A standardized method for LSP delineation was devised by Yi et al for 15 patients treated with IMRT for rectal or anal cancers. ${ }^{6}$ However, their study was criticized for its small cohort sample size and short follow-up period. Recently, Min et al found the Yi et al LSP contouring protocol to be a useful and reproducible guideline tool; however,
Radiation Oncology, Comprehensive Cancer Center, King Fahad Medical City, PO Box 59046, Riyadh 59046, Saudi Arabia

Tel +966 I 2889999

Fax +966 I 46I 4006

Email mkhairuddin@kfmc.med.sa 
they proposed the delineation of lumbosacral plexus regions (LSPRs), the regions in which LSP is likely to be present if it is radiologically invisible. ${ }^{7}$

In the present study, we aimed to delineate LSP and evaluate both dose distribution within LSP and its correlation with RILSP.

\section{Materials and methods}

After receiving approval from our institutional review board, we chose our study participants from among patients with cervical cancer who were treated at our institute between August 2007 and July 2012, using a whole-pelvis concurrent chemoradiation with IMRT technique (using six or eight coplanar beams), followed by image-guided conformal high-dose-rate brachytherapy (BT). Only those patients who met the following eligibility criteria were selected: histologically proven cervical cancer; FIGO stage IIB-IIIB, but with no evidence for distant metastasis; had undergone radical whole-pelvis concurrent chemoradiation, using the IMRT technique, followed by high-dose-rate BT; no locoregional or distant failure (disease-free); and a minimal follow-up period of at least 24 months. Patients who underwent adjuvant hysterectomy or had uncontrolled diabetes or dual-energy X-ray absorptiometry-confirmed severe osteopenia/osteoporosis were excluded.

\section{Lumbosacral plexus delineation}

For the purpose of study, LSP was delineated in each patient from the L4-L5 interspace (junction of L4 and L5 vertebrae) to the level of the sciatic nerve on contrast-enhanced treatment planning axial slices of $5 \mathrm{~mm}$ thickness by a radiation oncologist with assistance of a neuroradiologist using anatomy manuals and the Yi et al protocol. ${ }^{6}$ For radiologically visible LSP, a $5 \mathrm{~mm}$ diameter paint tool was used, and to the points of radiologically invisible LSP, a freehand tool was used to contour only the regions in which LSP was likely to be seen (LSP regions; Figure 1).

\section{Dose-volume histograms data}

For the purpose of study, the combined mean equivalent dose in $2 \mathrm{~Gy} /$ fraction $\left(\mathrm{EQD}_{2 \mathrm{IMRT}+\mathrm{BT}}\right)$ was calculated, and
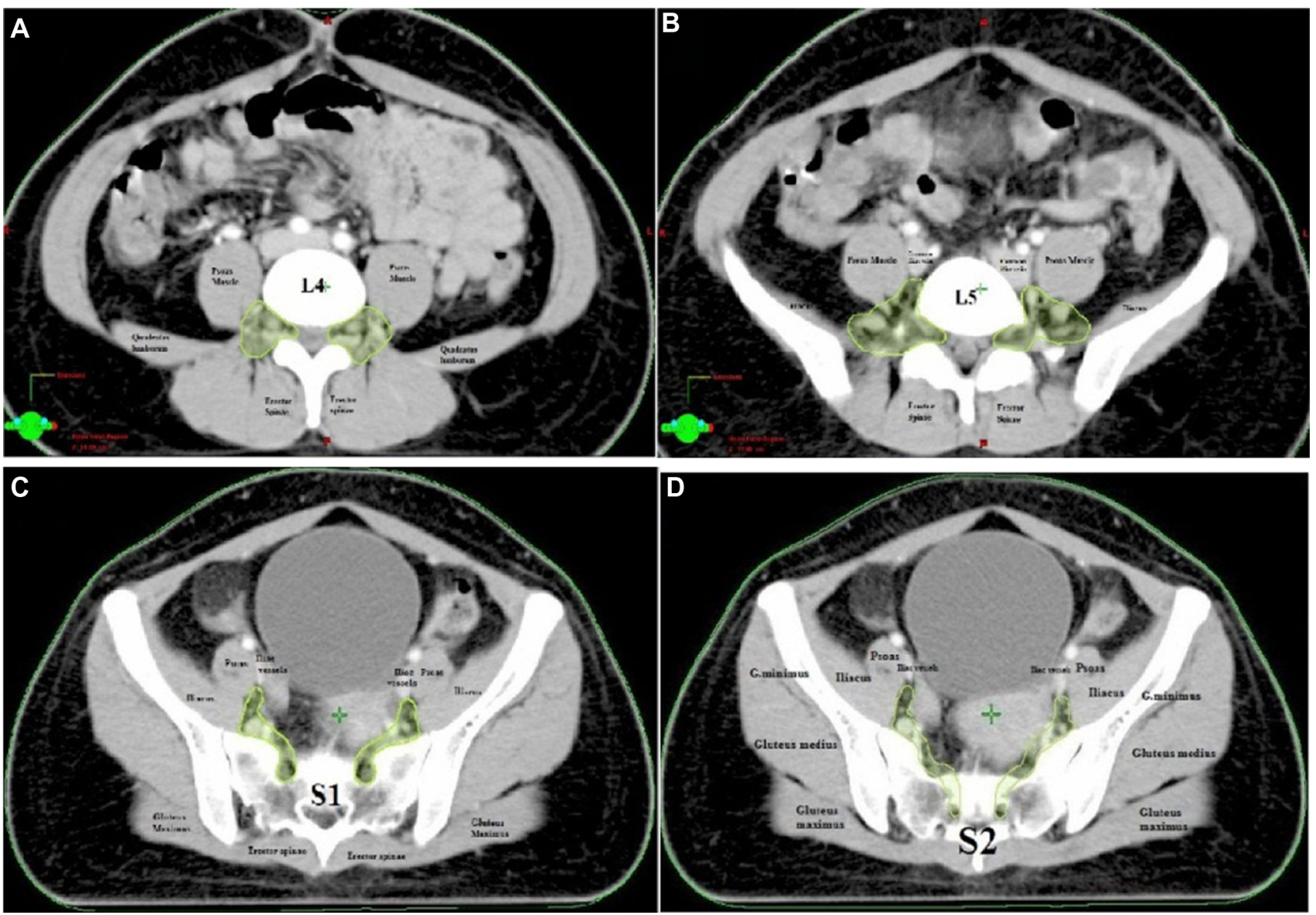

Figure I (Continued) 

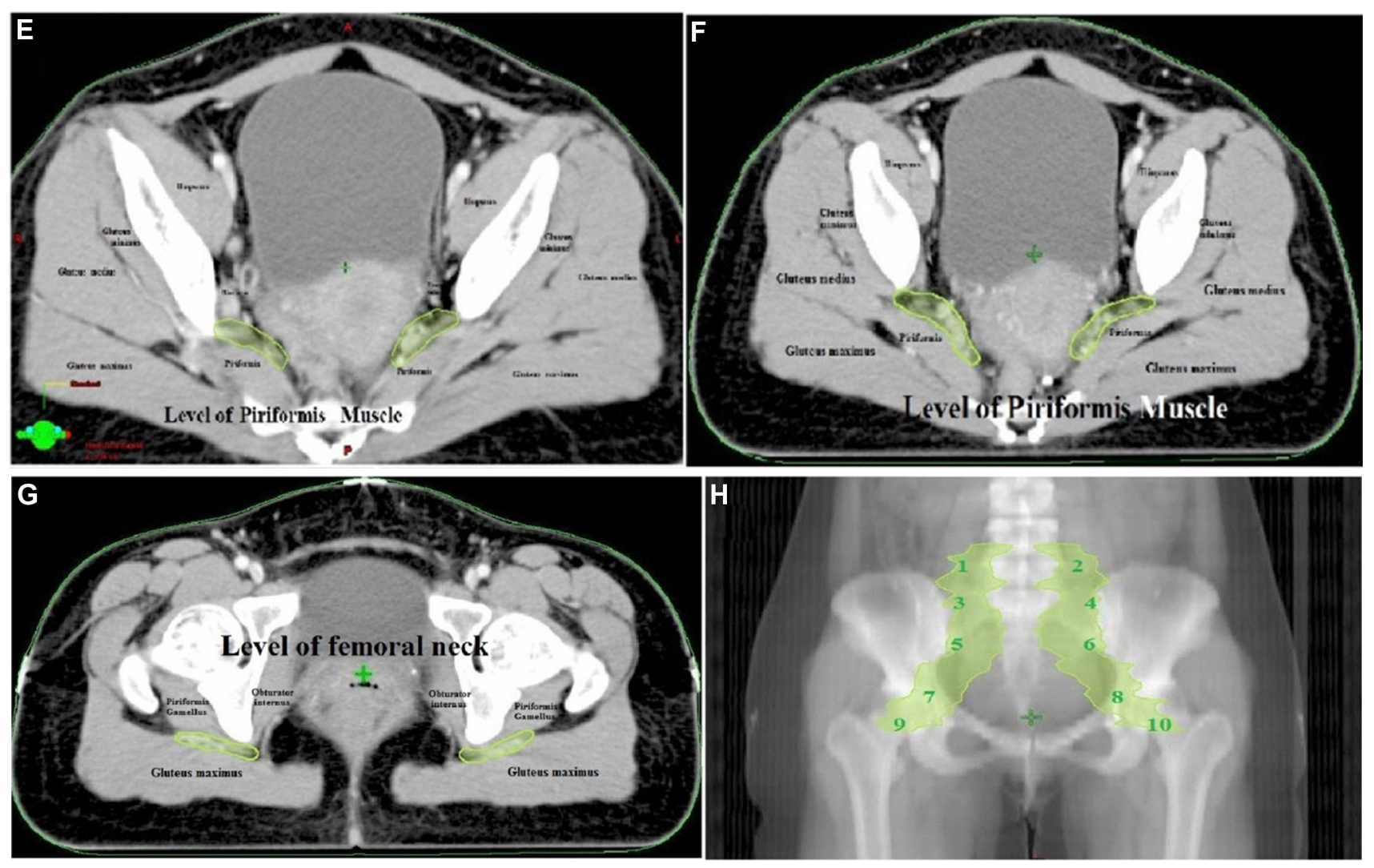

Figure I Cranial to piriformis portion of lumbosacral plexus (L4, L5, SI, and S2).

Notes: The areas shaded green indicate the lumbosacral plexus. (A-D) Prepiriformis portion of lumbosacral plexus (anterior to piriformis and posterior to internal obturator muscle). (E and $\mathbf{F}$ ) Postpiriformis portion of lumbosacral plexus at level of femoral neck (between gluteus maximus and obturator internus muscles). ( $\mathbf{G}$ and $\mathbf{H}$ ) Point doses calculation on the right and left lumbosacral plexus on digitally reconstructed radiographs.

based on each patient's dose-volume histogram (DVH), the total LSP volume, mean maximum dose to whole LSP, and volume percentages of the LSP absorbing, respectively, $5,10,20,30,40,50,55$, and 60 Gy (V5,V10,V20,V30, V40,V50,V55,V60) were then estimated. The continuous variables were dichotomized at their median values. In addition, point doses were calculated on LSP as P1 and P2 (point doses at the right and left portion of LSP at the level of the L4/L5 interspace), P3 and P4 (point doses at the right and left portion of LSP at the level of interspace L5/S1), P5 and P6 (point doses at the right and left portion of LSP at the level of the inferior part of the sacroiliac joint), P7 and P8 (point doses at the right and left portion of LSP at the level of ischial spine/acetabulum), and $\mathrm{P} 9$ and 10 (point doses at the right and left portion of LSP at the levels of femoral neck; Figure 1H).

\section{Evaluation of RILSP}

All patients were evaluated by a neurologist. A detailed history was obtained, and neurological examination was done to exclude other potential etiologies of lumbosacral plexopathy, along with magnetic resonance imaging (MRI). Additional positron emission tomography and needle electromyography were performed if suggested by the neurologist. The RILSP was defined as the "occurrence of paresthesias, numbness, dysesthesias, pain or lower extremity weakness confirmed on T2-weighted MRI (diffuse marrow and perineural foramina hyper-intensity)". The time of onset of RILSP was defined as the interval between the end of concurrent chemoradiation and the occurrence of the first RILSP symptom. Grading of RILSP was defined as follows: 1 is asymptomatic; 2 is symptomatic, but not interfering with activities of daily life; 3 is symptomatic and interfering with activities of daily life; and 4 is symptomatic with disability.

\section{Statistical analysis}

Associations between RILSP with relevant clinical data (age, comorbidities, FIGO stage, chemotherapy) and dosimetric data (total LSP volume, LSP mean dose $\left[D_{\text {mean }}\right]$, LSP maximum dose $\left[D_{\text {max }}\right], \mathrm{V} 5, \mathrm{~V} 10, \mathrm{~V} 20, \mathrm{~V} 30, \mathrm{~V} 40, \mathrm{~V} 50, \mathrm{~V} 55$, and $\mathrm{V} 60$, and point doses [P1, P2, P3, P4, P5, P6, P7, P8, P9, and P10]) were tested by Fisher exact test. Dosimetric data 
comparison between patients with RILSP and without RILSP (control patients) was done by using the Student's unpaired $t$-test. A $P$-value lower than 0.05 was considered statistically significant. All analysis was done using SPSS version 17.0 and Mathematica 9 software systems.

\section{Results}

Clinicopathologic and treatment characteristics of the 50 patients are summarized in Table 1. Median follow-up was 60 months (range, 24.1-65.4 months). All patients were treated by IMRT to total prescribed doses covering $95 \%$ of the planned target volume, ranging between $50.4 \mathrm{~Gy}$ and $59.0 \mathrm{~Gy}$ (median, 54 Gy) in 1.8 Gy fractions, followed by imageguided high-dose-rate BT $21 \mathrm{~Gy}$ in three sessions. The

Table I Clinicopathological and treatment characteristics

\begin{tabular}{ll}
\hline Variables & $\mathbf{N}(\%)$ \\
\hline Age & 46.2 years (range, 33-55) \\
ECOG performance scale, range & $0-2$ \\
Comorbidities & \\
Hypertension & \\
$\quad$ Yes & $5(10.0 \%)$ \\
$\quad$ No & $45(90.0 \%)$ \\
Diabetes & $3(6.0 \%)$ \\
$\quad$ Yes & $47(94.0 \%)$ \\
$\quad$ No & \\
Histopathology & $41(82.0 \%)$ \\
Squamous cell carcinoma & $8(16.0 \%)$ \\
Adenocarcinoma & $1(2.0 \%)$ \\
Adenosquamous cell carcinoma & \\
FIGO staging & $38(76.0 \%)$ \\
IIB & $6(12.0 \%)$ \\
IIIA & $6(12.0 \%)$ \\
IIIB & \\
Radiological primary tumor size & $17(34.0 \%)$ \\
$<5$ cm & $33(66.0 \%)$ \\
$>5$ cm &
\end{tabular}

Abbreviations: ECOG, European Cooperative Oncology Group; FIGO, International Federation of Gynecologists and Obstetricians; MRI, magnetic resonance imaging; EBRT, external beam radiation therapy; LN, lymph node.
$\mathrm{EQD}_{2 \text { IMRT+BT }}$ were 80.9 Gy (standard deviation, $\pm 7.2 \mathrm{~Gy}$ ). All patients received concurrent chemotherapy consisting of weekly cisplatin $40 \mathrm{mg} / \mathrm{m}^{2}$ (total dose not exceeding $70 \mathrm{mg}$ ). There was no significant interobserver or interoperational variation when the LSP was contoured.

\section{LSP DVH data}

Mean LSP volume was $93.2 \mathrm{~cm}^{3}$ (range, 72.3-117.3 $\mathrm{cm}^{3}$ ). The mean dose $\left(D_{\text {mean }}\right)$ and mean maximal dose $\left(D_{\max }\right)$ to the LSP were 47.9 Gy (range, 30.9-56.9 Gy) and 56.7 Gy (range, 54.6-62.7 Gy). The mean volume percentages of the LSP absorbing, respectively, 5, 10, 20, 30, 40, 50, 55, and 60 Gy were then calculated (V5, V10, V20, V30, V40, V50, $\mathrm{V} 55$, and V60), at $99.35 \%, 97.8 \%, 91.8 \%, 76.15 \%, 57.3 \%$, $36.05 \%, 4.15 \%$, and $1.7 \%$, respectively. Forty-eight patients $(96 \%)$ and 44 patients (88\%) received doses to the LSP in excess of $40 \mathrm{~Gy}$ or more and $50 \mathrm{~Gy}$, respectively. Twelve (24\%) and $5(10 \%)$ patients received doses to LSP in excess of $55 \mathrm{~Gy}$, respectively. The cumulative LSP DVHs for all patients are shown in Figure 2. The points P5, P6, P7, and P8 absorbed the highest doses when compared with other points, as shown in box plot Figure 3 .

\section{RILSP and correlation with DVH data}

Among 50 patients, four ( $8 \%$ ) were found to have a clinicoradiologically confirmed diagnosis of RILSP of grade 2 and 3 without any evidence of tumor recurrence. The initial time for onset of RILSP from the completion of treatment was 20 months (Table 2). In patients with RILSP, the $D_{\text {mean }}, D_{\max }$,

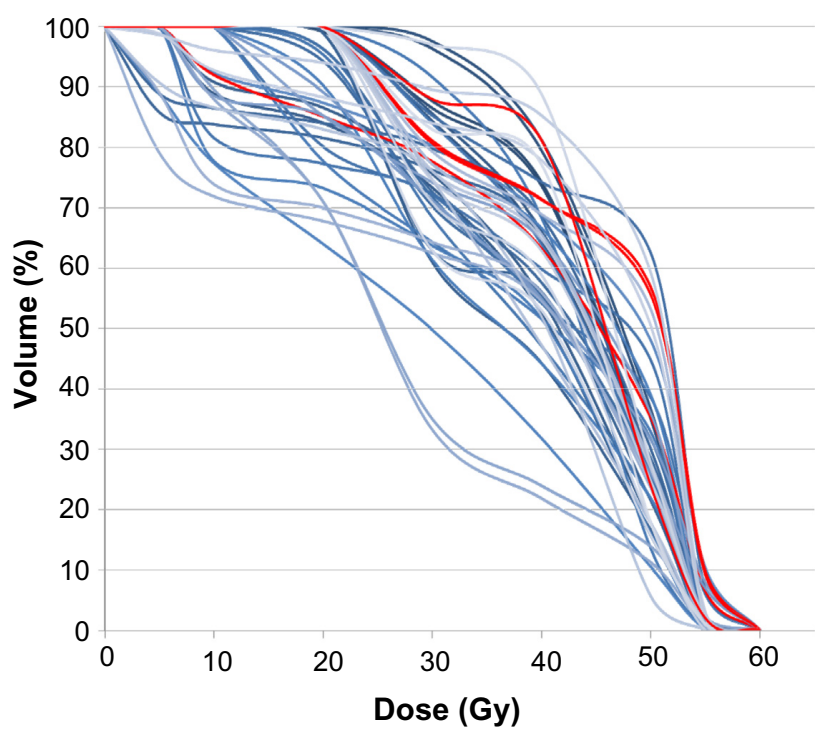

Figure 2 Cumulative lumbosacral plexus dose-volume histograms of all patients (red lines) on dose-volume histogram, showing patients in whom radiation-induced lumbosacral plexopathy was observed. 


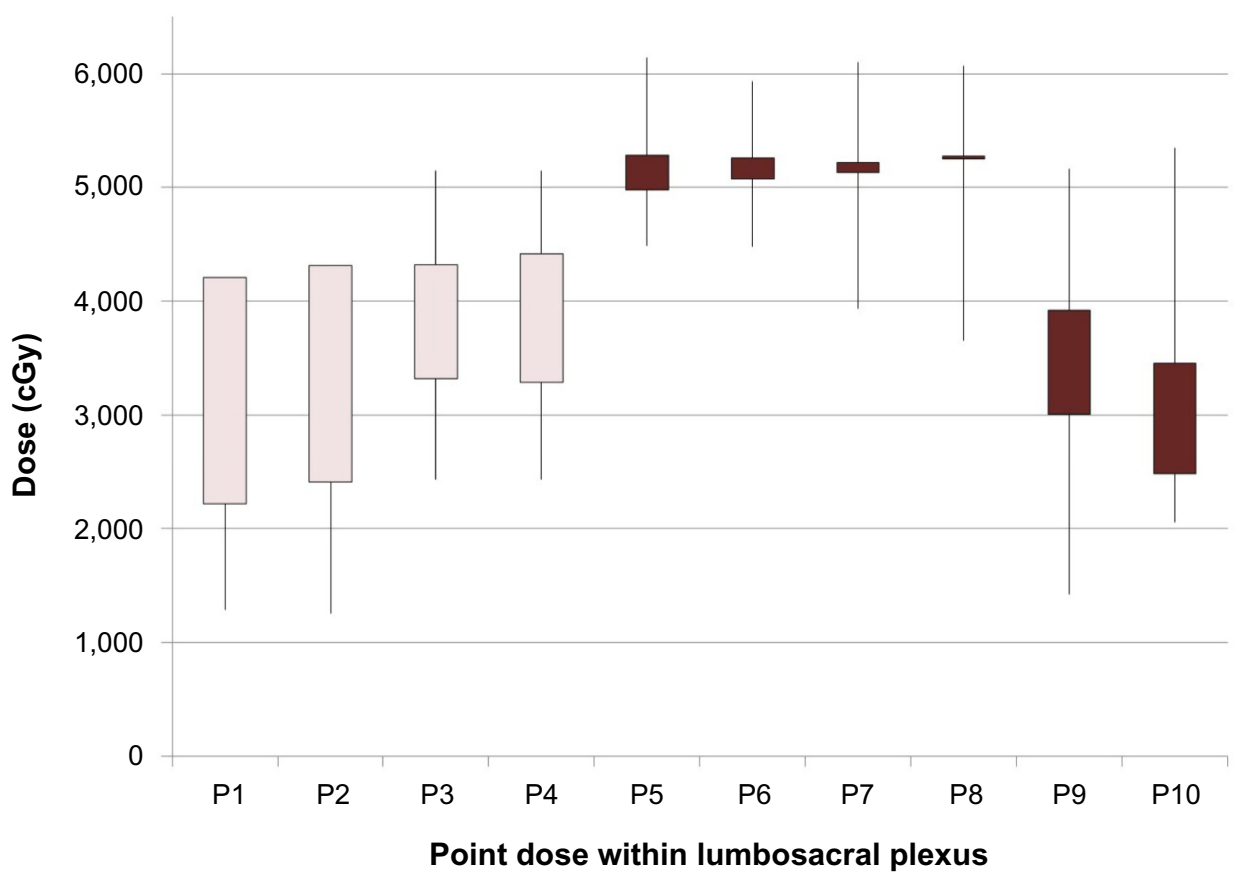

Figure 3 Box plot of the absorbed doses at points I, 2, 3, 4, 5, 6, 7, 8, 9, and 10 .

V40, V50, V55, and V60 were 52.9 Gy, 59.6 cGy, 61.8\% (range, 55\%-64.3\%), 44.4\% (range, 36\%-53\%), 8\% (range, $6.1 \%-10.2 \%$ ), and $1.8 \%$ (range, $1.0 \%-2.3 \%$ ), respectively. Statistically significant associations are presented in Table 3, where the $D_{\text {mean }}, D_{\text {max }}, \mathrm{V} 40, \mathrm{~V} 50, \mathrm{~V} 55$, and V60 of LSP were associated with a higher number of RILSP, with $P$-values of 0.03, 0.04, 0.04, 0.01, 0.001, and 0.001, respectively. Point doses (P5, P6, P7, and P8) were also found to be significantly associated with RILSP $(P=0.02)$.

\section{Discussion}

RILSP is rare, but is one of the most disabling complications of pelvic radiation therapy, as the leg weakness or incapacitating pain immobilize the patient, leading to infection, deep venous thrombosis, and poor quality of life. ${ }^{8}$ However, the true prevalence of RILSP in patients with cervical cancer treated with IMRT is underestimated, as the LSP is not routinely sought during target delineation in such cases. Differential diagnosis of RILP is always not easy; however, some clinical features may be useful. For example, in tumor/metastatic LSP, the pain may be relieved to a certain extent by either lying on one side with the knees flexed or flexing the affected extremity at the hip in bed. In contrast, pain is not relieved with positioning in patients with RILSP. ${ }^{9}$ Neurological findings are unilateral in patients with tumor/metastatic LSP, and bilateral in RILSP. ${ }^{10}$ Autonomic involvement or sphincter disturbance is unusual in patients with RILSP. ${ }^{11}$ There is also the negative reverse straight leg raising test in RILSP. ${ }^{3}$ Finally, no definite relief of pain is seen in patients with RILSP after an intravenous bolus of $100 \mathrm{mg}$ dexamethasone. ${ }^{9}$ Patients with RILSP have usually unremarkable computed tomography scans, and MRI is considered the most accurate diagnostic tool for RILSP. ${ }^{12}$ Positron emission tomography (rule out tumor/metastatic LSP) and electromyography (low-amplitude motor unit potentials in RILSP) are helpful adjunct tools, along with MRI. ${ }^{13,14}$ To the best of our knowledge, this is the first study that has sought LSP contouring and its correlation with RILSP in patients with cervical cancer (Table 4).

Table 2 Characteristics of patients with radiation-induced lumbosacral plexopathy

\begin{tabular}{lllllll}
\hline Patient & $\begin{array}{l}\text { Age, } \\
\text { years }\end{array}$ & Stage & $\begin{array}{l}\text { Onset of } \\
\text { RILSP, months }\end{array}$ & Grade & Diagnostic tool & Treatment \\
\hline I & 44 & IIIA & 20 & 2 & MRI, PET & NSAIDs, TCA, AEDs, PT \\
2 & 52 & IIB & 43 & 2 & MRI, PET, EMG & NSAIDs, TCA, AEDs, PT \\
3 & 49 & IIB & 52 & 2 & MRI, PET & NSAIDs, TCA, AEDs, PT \\
4 & 55 & IIB & 52 & 3 & MRI, PET, EMG & NSAIDs, TCA, AEDs, \\
& & & & & & epidural steroid injections, PT \\
\hline
\end{tabular}

Abbreviations: MRI, magnetic resonance imaging; PET, positron imaging tomography; NSAIDs, nonsteroidal anti-inflammatory drugs; TCA, tricyclic antidepressants; AEDs, antiepileptic drugs; PT, physical therapy; EMG, electromyography. 
Table 3 Associations between age, lumbosacral volume, and its dose distribution and radiation-induced lumbosacral plexopathy (RILSP)

\begin{tabular}{|c|c|c|c|}
\hline Variables & Patients without LSP $(n=46)$ & Patients with LSP $(n=4)$ & $P$-value \\
\hline Age & 46.2 years (range, $33-53$ ) & 50 years (range, $47-55$ ) & 0.06 \\
\hline Diabetes & I (2\%) & $2(4 \%)$ & 0.05 \\
\hline LSP volume, $\mathrm{cm}^{3}$ & 92.97 (range, 72.3-115) & 93.56 (range, 74.0-II7.3) & 0.9 \\
\hline Mean doses to LSP & $42.95 \mathrm{~Gy}$ & $52.90 \mathrm{~Gy}$ & 0.03 \\
\hline Maximum doses to LSP, mean & $53.9 \mathrm{cGy}$ & $59.6 \mathrm{cGy}$ & 0.04 \\
\hline V5 & $98.7 \%$ (range, 79.1-100) & $100 \%$ & 0.9 \\
\hline V10 & $95.6 \%$ (range, $71.8-100)$ & $100 \%$ & 0.8 \\
\hline $\mathrm{V} 20$ & $91.1 \%$ (range, 64.0-100) & $92.5 \%$ (range, $70 \%-100 \%$ ) & 0.9 \\
\hline V30 & $75.1 \%$ (range, 33.2-97.6) & $77.2 \%$ (range, 64.3\%-91.6\%) & 0.7 \\
\hline V40 & $52.8 \%$ (range, 21.9-62.8) & $61.8 \%$ (range, $55.0 \%-64.3 \%$ ) & 0.01 \\
\hline V50 & $27.7 \%$ (range, 10.6-49.0) & $44.4 \%$ (range, $36.0 \%-53.0 \%$ ) & 0.001 \\
\hline V55 & $0.31 \%$ (range, $0.0-1.7 I)$ & $8.0 \%$ (range, $6.1 \%-10.2 \%$ ) & 0.001 \\
\hline V60 & 0 & I.8\% (range, I.0\%-2.3\%) & 0.001 \\
\hline
\end{tabular}

Abbreviation: LSP, lumbosacral plexus.

In present study, the incidence of RILSP (8\%) in cervical cancer is much higher than that reported in the literature $(\sim 1.3 \%-6.67 \%))^{4,6}$ However, none of the patients in present study had grade 3 (disabling) RILSP. The possible explanation for high incidence of RILSP in our cohort can be explained by the fact that previous reports were mainly retrospective, case reports/series, with low sample size and nongynecologic tumors, and were mostly from the radiotherapy-alone era. When using radiotherapy alone with or without $\mathrm{BT}$, the tolerance to the LSP (tolerance dose; $5 \%$ probability of severe sequelae in 5 years) has been estimated at 47 and $60 \mathrm{~Gy}$, respectively; RILSP was estimated at $70-80$ Gy for full-volume irradiation. ${ }^{15,16}$ However, the radiosensitivity of peripheral nerves is likely enhanced by concomitant chemotherapy, and the RILSP has been reported at much lower doses (50-60 Gy), as seen in the present study, making LSP an organ at risk during IMRT planning in such patients. ${ }^{17}$ In our study, LSP volumes (mean volume, $93.2 \mathrm{~cm}^{3}$ ) were consistent with those described by Yi et al (mean volume, $100 \mathrm{~cm}^{3}$ ), which also validated this study; however, it is larger than those described by Min et al (mean volume, 40.9-58.4 $\left.\mathrm{cm}^{3}\right),{ }^{6,7}$ The reason for larger volumes can be explained by the contouring of LSPRs when LSP was radiologically invisible in our series. Further in our study, the mean V50, V55, and V60 were slightly higher (ie, $36 \%$ versus $22 \%$; $4.15 \%$ versus $0.5 \%$; and $1.7 \%$ versus $0 \%$ ), which can be explained by different IMRT techniques, different concurrent chemotherapy, parametrial boost in patients with cervical cancer, and higher point doses to P5, P6, P7, and P8. In the present study, we also noticed that risk for RILSP is more in patients older than 50 years of age and in those who are diabetic, although this difference was not significantly high in our cohort. In addition, the mean LSP dose (42.95 Gy) and mean V40 (52.8\%), V50 (27.7\%), V55 (0.31\%), and V60 (0\%) in our patients without RILSP indicates that mean dose lower than $45 \mathrm{~Gy}$, V40 lower than $55 \%$, V50 lower than $30 \%$, V55 lower than 5\%, and V60 lower than $0.5 \%$ for LSP during IMRT planning (especially focusing at the levels of P5, P6, P7, and P8) can significantly reduce the risk for RILSP, ${ }^{7}$ as treatment for RILSP is usually symptomatic and often refractory.

Strengths of our study are that it was the first study to mention RILSP and dosimetric data correlation in patients with cervical cancer and its appropriate selection of patients,

Table 4 Studies reporting the lumbosacral plexus delineation, dosimetric data, and its correlation with RILSP

\begin{tabular}{|c|c|c|c|c|c|c|c|c|}
\hline Study & Patients & Malignancy & LSP volume & $\begin{array}{l}\text { Mean maximal } \\
\text { dose to the } \\
\text { LSP, Gy }\end{array}$ & V40, \% & V50, \% & V55, \% & $\begin{array}{l}\text { Incidence of RILSP } \\
\text { and initial onset } \\
\text { after treatment }\end{array}$ \\
\hline Yi et al ${ }^{6}$ & 15 & Rectal/anal cancer & $\begin{array}{l}100 \pm 22 \mathrm{~cm}^{3} \\
(71-138)\end{array}$ & $\begin{array}{l}52.6 \\
(44.5-58.6)\end{array}$ & $58 \pm 19$ & $22 \pm 23$ & $0.5 \pm 0.9$ & $7 \%, 13$ months \\
\hline Min et $\mathrm{al}^{7}$ & 10 & $\begin{array}{l}\text { Cervical/endometrial/ } \\
\text { rectal/anal cancer }\end{array}$ & $40.9-58.4 \mathrm{~cm}^{3}$ & 52.2 & $\begin{array}{l}57.83 \\
(30-74.5)\end{array}$ & $\begin{array}{l}16.86 \\
(0-55.3)\end{array}$ & $\begin{array}{l}1.23 \\
(0-\mid 1.2)\end{array}$ & None \\
\hline $\begin{array}{l}\text { Present } \\
\text { study }\end{array}$ & 50 & Cervical cancer & $\begin{array}{l}93.2 \mathrm{~cm}^{3} \\
(72.3-117.3)\end{array}$ & $\begin{array}{l}56.7 \\
(54.6-62.7)\end{array}$ & $\begin{array}{l}61.8 \\
(55-64.3)\end{array}$ & $\begin{array}{l}44.4 \\
(36-53)\end{array}$ & $\begin{array}{l}8 \\
(6.1-10.2)\end{array}$ & $8 \%, 20$ months \\
\hline
\end{tabular}

Abbreviations: LSP, lumbosacral plexus; RILSP, radiation-induced lumbosacral plexopathy. 
periodic neurological assessment, and long follow-up. However, our study can be criticized for its low sample size and shorter follow-up.

\section{Conclusion}

RILSP in IMRT with concurrent chemotherapy in cervical cancer still represents a clinically underestimated problem, and LSP delineation is not yet performed routinely, probably because of limited literature data. The mean dose lower than 45 Gy, V40 lower than 55\%, V50 lower than 30\%, V55 lower than $5 \%$, and V60 lower than $0.5 \%$ for LSP during IMRT planning (especially focusing at levels of P5, P6, P7, and P8) can reduce the risk for RILSP. However, further large prospective studies on dose-RILSP in patients with cervical cancer are therefore warranted, and LSP should be considered as an organ at risk in all patients with cervical cancer who are receiving IMRT.

\section{Disclosure}

The authors report no conflicts of interest in this work.

\section{References}

1. Dahele M, Davey P, Reingold S, Shun Wong C. Radiation-induced lumbo-sacral plexopathy (RILSP): an important enigma. Clin Oncol (R Coll Radiol). 2006;18(5):427-428.

2. Topikan E, Onal HC, Yavuz AA, Yavuz MN. Pathophysiology and management of radiation-induced lumbosacral plexopathy. Turk Onkoloji Dergisi. 2008;23:147-152.

3. Saphner T, Gallion HH, Van Nagell JR, Kryscio R, Patchell RA. Neurologic complications of cervical cancer. A review of 2261 cases. Cancer. 1989;64(5):1147-1151.

4. Georgiou A, Grigsby PW, Perez CA. Radiation induced lumbosacral plexopathy in gynecologic tumors: clinical findings and dosimetric analysis. Int J Radiat Oncol Biol Phys. 1993;26(3):479-482.
5. Galvin JM, Ezzell G, Eisbrauch A, et al; American Society for Therapeutic Radiology and Oncology; American Association of Physicists in Medicine. Implementing IMRT in clinical practice: a joint document of the American Society for Therapeutic Radiology and Oncology and the American Association of Physicists in Medicine. Int J Radiat Oncol Biol Phys. 2004;58(5):1616-1634.

6. Yi SK, Mak W, Yang CC, et al. Development of a standardized method for contouring the lumbosacral plexus: a preliminary dosimetric analysis of this organ at risk among 15 patients treated with intensity-modulated radiotherapy for lower gastrointestinal cancers and the incidence of radiation-induced lumbosacral plexopathy. Int $J$ Radiat Oncol Biol Phys. 2012;84(2):376-382.

7. Min M, Roos D, Keating E, et al. External validation of the lumbosacral plexus-contouring protocol developed by Yi etal. (IJROBP 2012;84: 376-382) for pelvic malignancies. J Med Imaging Radiat Oncol. 2014;58(1):117-124.

8. Pettigrew LC, Glass JP, Maor M, Zornoza J. Diagnosis and treatment of lumbosacral plexopathies in patients with cancer. Arch Neurol. 1984;41(12):1282-1285.

9. Thomas JE, Cascino TL, Earle JD. Differential diagnosis between radiation and tumor plexopathy of the pelvis. Neurology. 1985;35(1):1-7.

10. Jaeckle KA, Young DF, Foley KM. The natural history of lumbosacral plexopathy in cancer. Neurology. 1985;35(1):8-15.

11. Jaeckle KA. Neurological manifestations of neoplastic and radiationinduced plexopathies. Semin Neurol. 2004;24(4):385-393.

12. Planner AC, Donaghy M, Moore NR. Causes of lumbosacral plexopathy. Clin Radiol. 2006;61(12):987-995.

13. Brejt N, Berry J, Nisbet A, Bloomfield D, Burkill G. Pelvic radiculopathies, lumbosacral plexopathies, and neuropathies in oncologic disease: a multidisciplinary approach to a diagnostic challenge. Cancer Imaging. 2013;13(4):591-601.

14. Tong HC. Specificity of needle electromyography for lumbar radiculopathy in 55- to 79-yr-old subjects with low back pain and sciatica without stenosis. Am J Phys Med Rehabil. 2011;90(3):233-238.

15. Emami B, Lyman J, Brown A, et al. Tolerance of normal tissue to therapeutic irradiation. Int J Radiat Oncol Biol Phys. 1991;21(1):109-122.

16. Stryker JA, Sommerville K, Perez R, Velkley DE. Sacral plexus injury after radiotherapy for carcinoma of cervix. Cancer. 1990;66(7): 1488-1492.

17. Klimek M, Kosobucki R, Luczyńska E, Bieda T, Urbański K. Radiotherapy-induced lumbosacral plexopathy in a patient with cervical cancer: a case report and literature review. Contemp Oncol (Pozn). 2012;16(2):194-196.
OncoTargets and Therapy

\section{Publish your work in this journal}

OncoTargets and Therapy is an international, peer-reviewed, open access journal focusing on the pathological basis of all cancers, potential targets for therapy and treatment protocols employed to improve the management of cancer patients. The journal also focuses on the impact of management programs and new therapeutic agents and protocols on

\section{Dovepress}

patient perspectives such as quality of life, adherence and satisfaction. The manuscript management system is completely online and includes a very quick and fair peer-review system, which is all easy to use. Visit http://www.dovepress.com/testimonials.php to read real quotes from published authors. 